\title{
Expression status of cyclase-associated protein 2 as a prognostic marker for human breast cancer
}

\author{
LIHUA XU $^{1,2^{*}}$, SIDA PENG $^{1 *}$, QUNAI HUANG $^{3}$, YU LIU $^{3}$, HUA JIANG $^{3}, \mathrm{XI} \mathrm{LI}^{3}$ and JIANI WANG ${ }^{3}$ \\ ${ }^{1}$ Department of Hematology and ${ }^{2}$ Guangdong Key Laboratory of Urology, The First Affiliated Hospital of \\ Guangzhou Medical University, Guangzhou, Guangdong 510230; ${ }^{3}$ Breast Cancer Center, \\ The Third Affiliated Hospital of Sun Yat-sen University, Guangzhou, Guangdong 510630, P.R. China
}

Received March 10, 2016; Accepted April 22, 2016

DOI: $10.3892 /$ or.2016.5051

\begin{abstract}
Cyclase-associated protein 2 (CAP2) protein is reported to be upregulated in hepatocellular carcinoma (HCC). However, data regarding its expression pattern and clinical relevance in breast cancer are unknown. The aim of this study was to investigate CAP2 expression and its prognostic significance in breast cancer. CAP2 expression at the mRNA and protein levels was examined by real-time quantitativepolymerase chain reaction and western blotting in 10 paired breast cancer tissues and adjacent normal tissues. The expression level of CAP2 protein in normal breast epithelial cells and breast cancer cell lines was quantified by western blotting. CAP2 protein expression was analyzed in paraffin-embedded breast cancer samples, paired adjacent non-tumor and normal breast tissues by immunohistochemical analysis. Statistical analyses were also performed to evaluate the clinicopathological significance of CAP2 expression. The results showed that the expression of CAP2 mRNA and protein was higher in breast cancer than that noted in the adjacent normal tissues in 10 paired samples. The expression level of CAP2 protein in breast cancer cell lines was higher than that in normal breast epithelial cells. In paraffin-embedded tissue samples, the expression of CAP2 was higher in breast cancer than that found in the adjacent non-cancerous tissues and normal breast tissues. Compared with the adjacent non-cancerous tissues, overexpression of CAP2 was detected in $29.4 \%$ (37/126) of the patients. Overexpression of CAP2 was significantly associated with progesterone receptor $(P R)$ expression $(\mathrm{p}<0.05)$, and decreased overall survival (OS) $(\mathrm{p}<0.05)$. In multivariate
\end{abstract}

Correspondence to: Ms. Jiani Wang or Dr Xi Li, Breast Cancer Center, The Third Affiliated Hospital of Sun Yat-sen University, 600 Tianhe Road, Guangzhou, Guangdong 510630, P.R. China

E-mail: turtle_w@163.com

E-mail:dr-lixi@21cn.com

*Contributed equally

Key words: cyclase-associated protein 2, overexpression, breast cancer, prognosis analysis, expression of CAP2 was an independent prognostic factor for OS [hazard ratio (HR), 4.821; 95\% confidence interval (CI), 2.442-9.518; $\mathrm{p}<0.001]$. CAP2 is upregulated in breast cancer and is associated with the expression of $\mathrm{PR}$ and patient survival. CAP2 may serve as a prognostic indicator for patients with breast cancer.

\section{Introduction}

Breast cancer is the leading cause of cancer morbidity and mortality in women worldwide. Based on early diagnosis and treatment, the 5-year survival for patients with breast cancer has made noticeable improvement. Breast cancer still accounts for $25 \%$ of all cancer cases and $15 \%$ of all cancer-related deaths among females (1). Although estrogen receptor (ER), progesterone receptor (PR) and human epidermal growth factor receptor 2 (HER2)/neu protein are useful biomarkers in breast cancer diagnosis and treatment, the discovery of new biomarkers related to breast cancer can help build a deeper and comprehensive understanding of this disease.

Adenylyl cyclase-associated protein (CAP) is an evolutionarily highly conserved protein in all eukaryotes including mammals and was first identified in yeast as a protein that regulates both the actin cytoskeleton and the Ras/cAMP pathway $(2,3)$. The actin cytoskeleton plays an important role in cellular functions such as morphogenesis, cytokinesis and cell migration. An aberrant actin cytoskeleton usually underlies oncogenesis and cancer metastasis (4,5). In mammals, cells have two CAP isoforms, CAP1 and 2. Compared with CAP1, CAP2 has a more restricted expression pattern and is found mainly in skeletal muscle, cardiac muscle, brain and skin (6). At present, there are few studies on the relationship between CAPs and tumors. CAP1 overexpression was found in hepatocellular carcinoma (HCC), breast and ovarian cancers (7-9). Shibata et al reported that CAP2 is upregulated in HCC and is related to multistage hepatocarcinogenesis (10). Masugi et al found that CAP2 overexpression is a novel prognostic marker in malignant melanoma and its expression appears to increase stepwise during tumor progression (11).

However, the clinical significance of CAP2 in breast cancer remains unclear. In this study, we examined the CAP2 expression in breast cancer cell lines and tissue samples, and revealed its clinicopathological and prognostic significance. 


\section{Materials and methods}

Cell lines. Human breast cancer cell lines, including MDA-MB-435, MDA-MB-453, MCF-7, T47D, and SK-BR-3 were cultured in Dulbecco's modified Eagle's medium (DMEM; Gibco, Grand Island, NY, USA) supplemented with $10 \%$ fetal calf serum (HyClone, Logan, UT, USA) at $37^{\circ} \mathrm{C}$ in $5 \% \mathrm{CO}_{2}$. The procedure for primary culture of human mammary epithelial cells (HMECs) is similar to that for human nasopharyngeal epithelial cells (12).

Patients and specimens. This study was conducted using a total of 126 paraffin-embedded primary breast cancer samples from patients who were histopathologically diagnosed and underwent curative resection at the Third Affiliated Hospital of Sun Yat-sen University between March 2001 and December 2012. None of the patients received any type of neoadjuvant therapy and all of them underwent curative surgery. The clinical information of these cases is summarized in Table I. The follow-up time of the breast cancer cohort ranged from 2 to 131 months, and the median follow-up time was 111 months. Of these 126 breast cancer patients, paired adjacent non-cancerous tissues (the adjacent non-cancerous tissue was defined as at least $2 \mathrm{~cm}$ distant from the edge of the tumor) were obtained from 30 patients. Twenty normal breast tissues were obtained from patients that underwent mammaplasty.

Clinicopathological classification and staging were determined according to the American Joint Committee on Cancer (AJCC, 7th edition) criteria. Patient consent to the use of these clinical specimens for research purposes was gained prior and the protocol was approved by the Institutional Research Ethics Committee of Sun Yat-sen University. Ten paired breast cancer and adjacent non-cancerous tissues were collected immediately after surgery for real-time PCR and western blotting.

Real-time quantitative-polymerase chain reaction (RT-PCR) analysis. Total RNA samples were extracted from cell lines and primary breast tumor materials using TRIzol reagent (Invitrogen, Carlsbad, CA, USA) according to the manufacturer's instructions. Extracted RNA was pretreated with RNase-free DNase. Two micrograms of RNA from each sample were used for cDNA synthesis. For the PCR amplification of CAP2 cDNA, an initial amplification step using CAP2-specific primers was performed with a denaturation at $95^{\circ} \mathrm{C}$ for $10 \mathrm{~min}$, followed by 28 denaturation cycles at $95^{\circ} \mathrm{C}$ for $60 \mathrm{sec}$, then primer annealing at $58^{\circ} \mathrm{C}$ for $30 \mathrm{sec}$, and then a primer extension phase at $72^{\circ} \mathrm{C}$ for $30 \mathrm{sec}$. Upon completion of these cycling steps, a final extension at $72^{\circ} \mathrm{C}$ for 5 min was carried out before the reaction mixture was stored at $4^{\circ} \mathrm{C}$. Then real-time PCR was performed to determine the fold increase of CAP2 mRNA in each of the breast tumor and paired normal breast tissues from the same patient. The primer sequences were as follows: CAP2 sense, 5'-GCCGCCTGGAGT CGCTGTC-3' and antisense, 5'-AAAACTCGGCCACCATA CTGTCCA-3'. GAPDH (sense, 5'-TGTTGCCATCAATG ACCCC-3' and antisense, 5'-CTCCACGACGTACTCAGC-3') was used as an internal control. The primers were designed by Primer Express Software v2.0 (Applied Biosystems).
Glyceraldehyde-3-phosphate dehydrogenase (GAPDH) was used as an internal control, and all experiments were performed in triplicate.

Western blotting. Cells at $70-80 \%$ confluency were washed twice by ice-cold phosphate-buffered saline (PBS) and then lysed on ice by radioimmunoprecipitation assay (RIPA; Cell Signaling Technology, Inc., Danvers, MA) buffer which contained complete protease inhibitor cocktail (Roche Applied Science, Mannheim, Germany). Fresh tissue samples were ground into powder in liquid nitrogen and lysed by SDS-PAGE sample buffer. A total of $20 \mu \mathrm{g}$ of protein samples was separated on $10.5 \%$ SDS polyacrylamide gels and then transferred to PVDF membranes (Immobilon-P; Millipore, Billerica, MA, USA). The membranes were then blocked with $5 \%$ fat-free milk in Tris-buffered saline with $0.1 \%$ Tween-20 (TBST) for $1 \mathrm{~h}$ at room temperature. PVDF membranes were incubated with anti-CAP2 antibody (1:1,000, 15865-1-AP; Proteintech Group, Inc., Rosemont, IL, USA) overnight at $4^{\circ} \mathrm{C}$, and then with horseradish peroxidase-conjugated goat anti-rabbit IgG (SC-2004; Santa Cruz Biotechnology, Inc.). CAP2 expression was detected by ECL Western Blotting detection reagent (Amersham/GE Healthcare Life Sciences) according to the manufacturer's instructions. GAPDH $(1: 1,000$; Proteintech Group, Inc.) was used as loading control.

Immunohistochemical (IHC) analysis. IHC staining was performed to study altered protein expression in 126 human breast cancer tissues, 30 paired adjacent non-cancerous tissues, and 20 normal breast tissues. Briefly, $4-\mu \mathrm{m}$-thick paraffin sections of the tissue were deparaffinized with xylene and rehydrated. Antigenic retrieval was performed by submerging the slides into EDTA antigenic retrieval buffer and microwaving. In order to quench endogenous peroxidase activity, the slides were treated with $3 \%$ hydrogen peroxide in methanol, and then incubated with $1 \%$ bovine serum albumin to block non-specific binding. After that, sections were incubated with anti-CAP2 rabbit polyclonal antibody (1:100, bs-1616R; Beijing Bioss Biosynthesis Biotechnology Co., Ltd.; Beijing, China) at $4^{\circ} \mathrm{C}$ overnight. Normal goat serum was used as a negative control. The tissue sections were incubated with a biotinylated anti-rabbit secondary antibody after being washed 3 times, followed by further incubation with streptavidin-horseradish peroxidase complex (both from Abcam). Slides were immersed in 3-amino-9-ethyl carbazole and then counterstained with $10 \%$ Mayer's hematoxylin, finally dehydrated and mounted in Crystal Mount.

As for evaluation of immunostaining, the degree of immunostaining was viewed and scored separately by two pathologists, who were blinded to the histopathological characteristics and patient information of the samples. Scores given by the two independent pathologists were averaged for further comparative evaluation of CAP2 expression. The intensity of CAP2 staining was graded according to the following criteria: 0 , no staining; 1 , weak staining (light yellow); 2 , moderate staining (yellow brown); 3 , strong staining (brown). The percentage of stained tumor cells was scored as follows: 0 , no positive tumor cells; $1,1-25 \%$ positive tumor cells; $2,26-50 \%$ positive tumor cells; $3,51-75 \%$ positive tumor cells; $4,>75 \%$ positive tumor cells. 

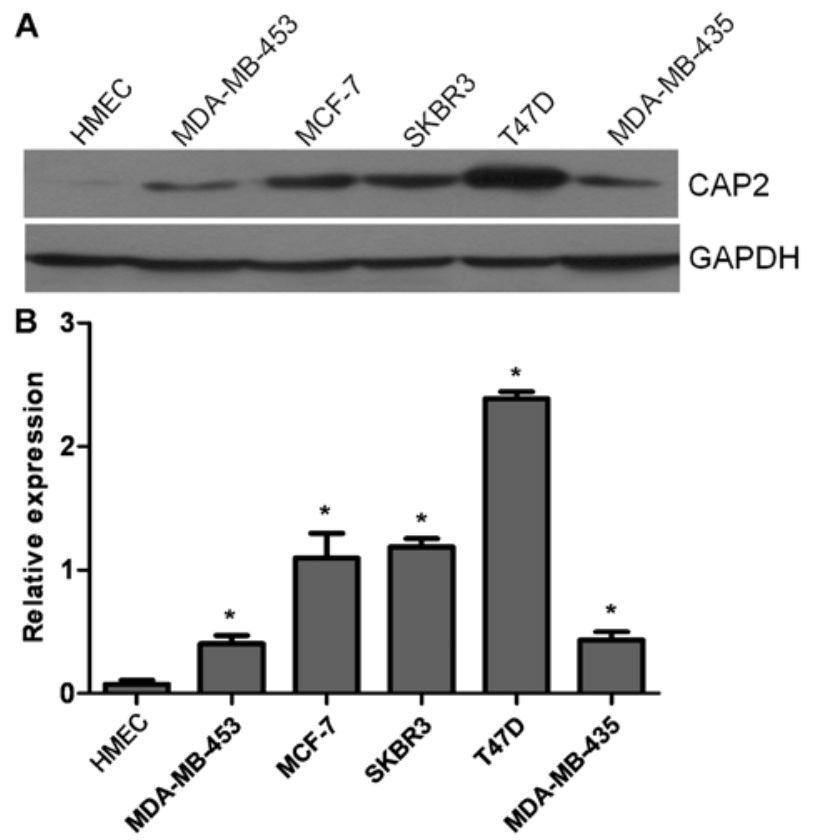

Figure 1. Overexpression of CAP2 protein in breast cancer cell lines and normal HMECs. (A) Expression of CAP2 protein in normal HMECs and breast cancer cell lines (MDA-MB-453, MCF-7, SK-BR-3, T47D, and MDA-MB-435) was examined by western blotting. Expression levels were normalized against GAPDH. (B) Quantitative analysis. Error bars represent the SD of the mean which was calculated from three parallel experiments. ${ }^{*} \mathrm{P}<0.05$. CAP2, cyclase-associated protein 2; HMECs, human mammary epithelial cells; GAPDH, glyceraldehyde-3-phosphate dehydrogenase; SD, standard deviation.

The staining score was calculated as the product of the proportion of positive tumor cells and the staining intensity score. The expression level of CAP2 was defined as follows: '-' (score 0, negative), '+' (score 1-4, weakly positive), '++' (score $5-8$, positive), ' +++ ' (score $9-12$, strongly positive). Cut-off values for CAP2 were chosen on the basis of the heterogeneity using log-rank test with respect to overall survival (OS). The optimal cut-off value was estimated as follows: a staining index score of $\geq 8$ was used to define tumors with high CAP2 expression and $<8$ indicated low CAP 2 expression.

Statistical analysis. The duration from the date of each patient's randomization to the date of death for any cause or the censoring of the patient at the last follow-up date was defined as OS. All the statistical analyses were conducted using the SPSS 20.0 statistical software packages. The differences in CAP2 expression between breast cancer tissues, adjacent non-cancerous tissues and normal breast tissues were analyzed by Chi-square test. Survival curves were plotted by Kaplan-Meier method and compared using the log-rank test. The relationship between CAP2 expression and other clinicopathological characteristics was analyzed by Chi-square test and Fisher's exact test. Bivariate correlations between the clinicopathological characteristics were calculated by Spearman's rank correlation coefficients. Clinicopathological characteristics used to predict prognosis in clinical practice were evaluated by univariate and multivariate Cox regression analyses. The chosen type of Cox model for univariate analysis was the enter method, and for multivariate analysis was

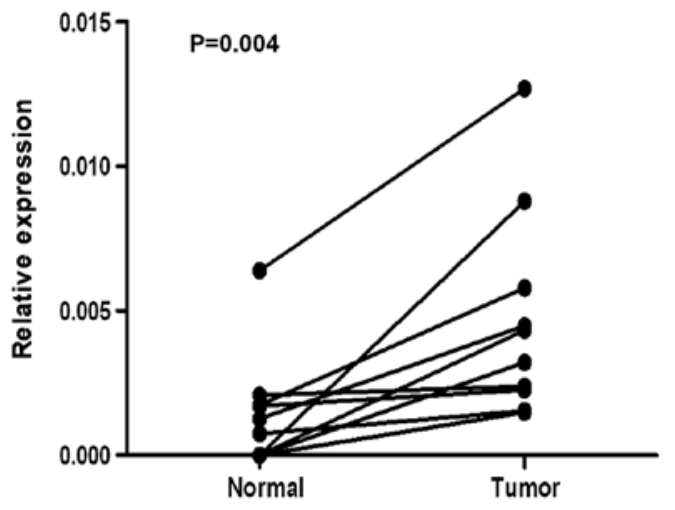

Figure 2. Expression levels of CAP2 mRNA in breast cancer and adjacent non-cancerous tissues. Expression levels of CAP2 mRNA in 10 paired breast cancer and adjacent non-cancerous tissues by real-time PCR. Normal, adjacent non-cancerous tissues; tumor, breast cancer tissues; CAP2, cyclase-associated protein 2 .

forward method. A p-value $<0.05$ was considered to indicate a statistically significant result.

\section{Results}

CAP2 is overexpressed in breast cancer cell lines. We used western blotting to evaluate the expression level of CAP2 protein in the breast cancer cell lines and normal HMECs. The expression levels of CAP2 were determined in five breast cancer cell lines (MDA-MB-453, MCF-7, SK-BR-3, T47D and MDA-MB-435) and were compared with CAP2 expression levels in primary cultured normal HMECs. CAP2 protein was highly expressed in all five breast cancer cell lines and only weakly expressed in the HMECs (Fig. 1A and B).

CAP2 is overexpressed in breast cancer tissues. To determine whether CAP2 is also highly expressed in human breast cancer samples, we performed RT-PCR and western blotting on 10 breast tumor samples and adjacent non-cancerous tissues. As illustrated in Fig. 2, CAP2 mRNA was expressed at higher levels in all of the 10 breast cancer tissues than that noted in the adjacent non-cancerous tissues, with the differential expression level ranging from 4.7- to 49.4-fold. Consistent with these data, CAP2 protein was also found to be upregulated in the fresh breast cancer tissues compared with that found in the adjacent non-cancerous tissues (Fig. 3). For immunostaining results, overexpression of CAP2 was observed in 29.37\% (37/126) of the breast cancer patients. CAP2 protein staining was weak or no staining was observed in the adjacent non-tumor tissues and normal breast tissues; only $6.67 \%(2 / 30)$ in the adjacent non-tumor tissues and 5\% (1/20) in normal breast tissues. The difference between the breast cancer group and the adjacent non-tumor group was statistically significant $\left(\chi^{2}=6.658, p=0.01\right)$. The difference between the breast cancer group and the normal breast tissue group was statistically significant $\left(\chi^{2}=5.322, p=0.01\right)$. But the difference between the adjacent non-tumor group and normal breast tissue group was not statistically significant $\left(\chi^{2}=0.059, \mathrm{p}=0.651\right)$.

CAP2 overexpression is associated with breast cancer clinical features. For better understanding of the potential 

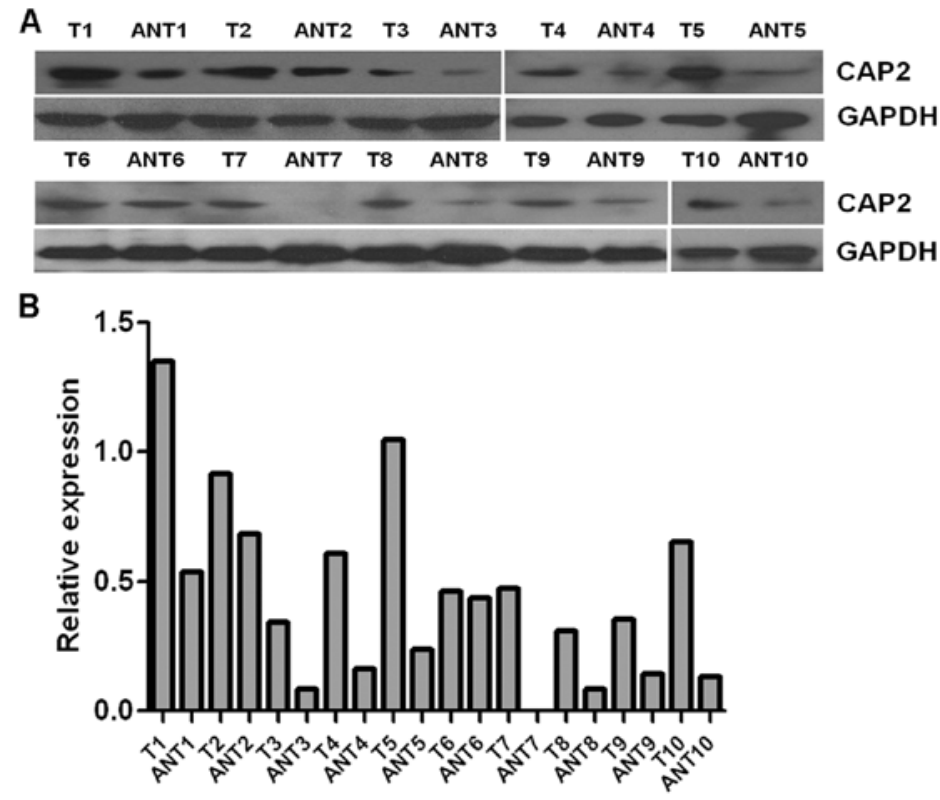

Figure 3. CAP2 protein expression in 10 paired breast cancer and adjacent non-cancerous tissues in patients with breast cancer. (A) Expression levels of CAP2 protein in 10 paired breast cancer and adjacent non-cancerous tissues by western blotting. (B) Quantitative analysis. T, breast cancer tissues; ANT, adjacent non-cancerous tissues; CAP2, cyclase-associated protein 2.
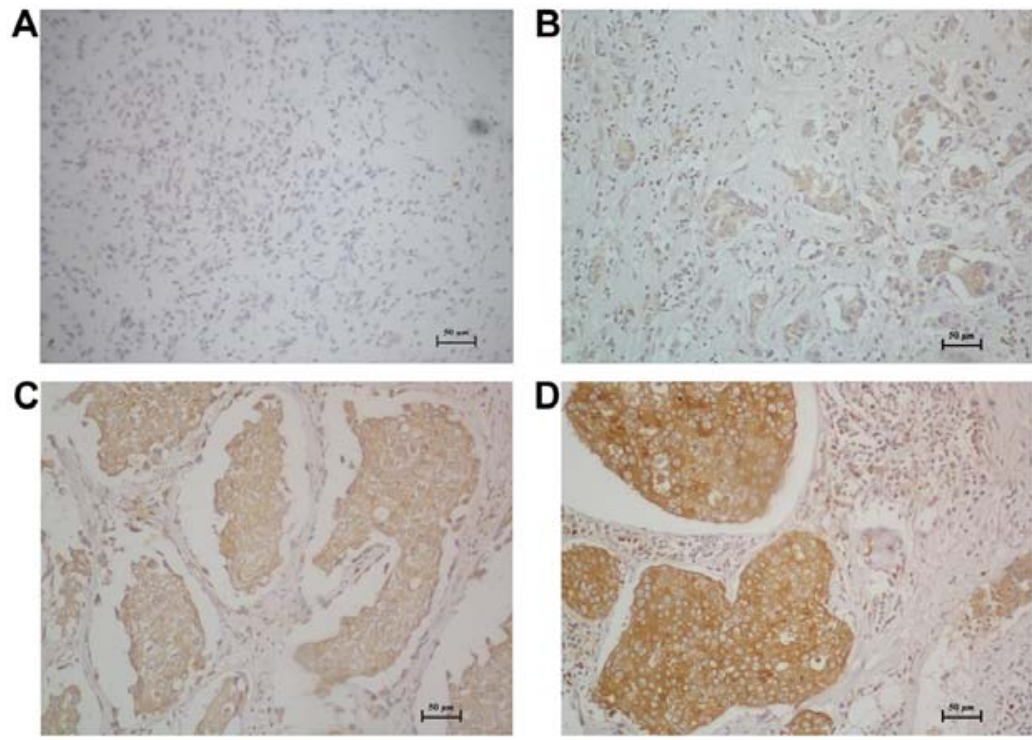

Figure 4. IHC analysis of CAP2 protein expression. CAP2 expression was mainly localized in the cytoplasm of breast tumor cells. CAP2 is weak or not expressed in normal breast epithelial cells. (A) Staining of CAP2 in normal breast tissues. (B) ' + ' expression (score 1-4, weakly positive), (C) '++' expression (score 5-8, positive), and (D) '+++' expression (score 9-12, strongly positive) of CAP2 in breast cancer tissues. IHC, immunohistochemical; CAP2, cyclase-associated protein 2 .

roles of CAP2 in breast cancer development and progression, we investigated the status of CAP2 expression in 126 paraffin-embedded archived breast cancer tissues by IHC staining, including 10 stage I, 76 stage II, and 40 stage III tumors. Among the 126 samples, high CAP2 protein expression was detected in 37 samples $(29.37 \%)$ and weak or no staining was observed in 89 tumor samples $(70.63 \%$, Table I). As shown in Fig. 4, CAP2 was highly expressed in breast cancer tissues. In contrast, no signals or only weak signals were detected in adjacent non-cancerous and normal breast tissues. The subcellular location of CAP2 was mainly at the cytoplasm.

We further analyzed the correlation between CAP2 expression and the clinicopathological characteristics of the breast cancer patients. As summarized in Table I, there were no significant correlations between the expression of CAP2 protein and patient age, clinical stage, $\mathrm{T}$ classification, $\mathrm{N}$ classification, differentiation, ER expression levels or HER2 in patients with breast cancer. However, the CAP2 expression was markedly associated with PR expression levels $(p=0.005)$. 
Table I. Correlation of CAP2 expression with clinicopathologic features of the breast cancer cases.

\begin{tabular}{|c|c|c|c|c|}
\hline \multirow[b]{2}{*}{ Characteristics } & \multirow{2}{*}{$\begin{array}{c}\text { Total } \\
(\mathrm{n}=126) \\
\mathrm{n}(\%)\end{array}$} & \multicolumn{2}{|c|}{ CAP2 expression } & \multirow[b]{2}{*}{ P-value } \\
\hline & & $\begin{array}{c}\text { Low }(\mathrm{n}=89) \\
\mathrm{n}(\%)\end{array}$ & $\begin{array}{c}\text { High }(\mathrm{n}=37) \\
\mathrm{n}(\%)\end{array}$ & \\
\hline Age (years) & & & & 0.626 \\
\hline$\geq 60$ & $37(29.37)$ & $25(67.6)$ & $12(32.4)$ & \\
\hline$<60$ & $89(70.63)$ & $64(71.9)$ & $25(28.1)$ & \\
\hline Clinical stage & & & & 0.223 \\
\hline I & $10(7.94)$ & $6(60.0)$ & $4(40.0)$ & \\
\hline II & $76(60.32)$ & $58(76.3)$ & $18(23.7)$ & \\
\hline III & $40(31.75)$ & $25(62.5)$ & $15(37.5)$ & \\
\hline $\mathrm{T}$ classification & & & & 0.506 \\
\hline $\mathrm{T} 1$ & $26(20.63)$ & $20(76.9)$ & $6(23.1)$ & \\
\hline $\mathrm{T} 2$ & $87(69.05)$ & $60(69.0)$ & $27(31.0)$ & \\
\hline $\mathrm{T} 3$ & $13(10.32)$ & $9(69.2)$ & $4(30.8)$ & \\
\hline $\mathrm{N}$ classification & & & & 0.588 \\
\hline N0 & $49(38.89)$ & $35(71.4)$ & $14(28.6)$ & \\
\hline N1 & $39(30.95)$ & $29(74.4)$ & $10(25.6)$ & \\
\hline $\mathrm{N} 2$ & $30(23.81)$ & $21(70.0)$ & $9(30.0)$ & \\
\hline N3 & $8(6.35)$ & $4(50.0)$ & $4(50.0)$ & \\
\hline Differentiation & & & & 0.448 \\
\hline Well & $13(10.32)$ & $11(84.6)$ & $2(15.4)$ & \\
\hline Moderate & $94(74.60)$ & $64(68.1)$ & $30(31.9)$ & \\
\hline Poor & $19(15.08)$ & $14(73.7)$ & $5(26.3)$ & \\
\hline ER expression & & & & 0.122 \\
\hline Negative & $45(35.71)$ & $28(62.2)$ & $17(37.8)$ & \\
\hline Positive & $81(64.29)$ & $61(75.3)$ & $20(24.7)$ & \\
\hline PR expression & & & & 0.005 \\
\hline Negative & $54(42.86)$ & $31(57.4)$ & $23(42.6)$ & \\
\hline Positive & $72(57.14)$ & $58(80.6)$ & $14(19.4)$ & \\
\hline HER 2 expression & & & & 0.536 \\
\hline Negative & $90(71.43)$ & $65(72.2)$ & $25(27.8)$ & \\
\hline Positive & $36(28.57)$ & $24(66.7)$ & $12(33.3)$ & \\
\hline
\end{tabular}

CAP2, cyclase-associated protein 2; ER, estrogen receptor; PR, progesterone receptor; HER2, human epidermal growth factor receptor 2.

Association between CAP2 expression and patient survival. Survival analysis showed a clear negative correlation between CAP2 protein expression level and the OS of patients with breast cancer ( $\mathrm{p}<0.001$, Fig. 5A). In addition, Cox regression revealed that CAP2 expression, clinical stage, and PR expression were independent prognostic factors for OS (Table II).

Furthermore, we analyzed the prognostic value of CAP2 in selective patient subgroups stratified by patient age, tumor grade, $\mathrm{T}$ and $\mathrm{N}$ classification, respectively. For patients $<60$ years of age, the expression of CAP2 was strongly associated with OS duration (Fig. 5C; log-rank test, $\mathrm{p}<0.001$ ), but not for patients $>60$ years of age (Fig. 5B; log-rank test, $\mathrm{p}=0.09$ ). The expression of CAP2 was strongly associated with OS duration of the patients with both early-stage tumors (stage I; log-rank test, $\mathrm{p}=0.01$ ) and late-stage tumors (stage II and III; log-rank test, p<0.001) (Fig. 5D and E). However, when it was evaluated according to $\mathrm{T}$ and $\mathrm{N}$ classification, the impact on the outcome associated with the expression of CAP2 continued to be more favorable only in T1-2 subgroups (Fig. 5F; log-rank test, $\mathrm{p}<0.001$ ) and N1-3 subgroup (Fig. 5I; log-rank test, $\mathrm{p}<0.001$ ) but not in the T3 subgroup (Fig. 5G; log-rank test, $\mathrm{p}=0.06$ ) and N0 subgroup (Fig. $\mathrm{H}$; log-rank test, $\mathrm{p}=0.119$ ).

\section{Discussion}

CAPs are conserved in all eukaryotes. CAP2 is part of the actin cytoskeleton, which regulates cell shape, cell motility and muscle contraction. The cytoskeleton is assembled by polymerization of globular actin (G-actin) monomers into filamentous actin (F-actin). The balance of F- and G-actin is coordinated by actin-binding proteins (13). There are two CAP homologs in mammals, CAP1 and CAP2. CAP1 is widely expressed in most cells and tissues, while CAP2 expression is restricted to the brain, skin, skeletal muscle, cardiac muscle and testis $(6,14)$. At the subcellular level, CAP2 is found in the cytoplasm although, unlike other isoforms, some CAP2 exists in the nucleus. It is likely that CAP1 and CAP2 complement each other in some cellular functions, but CAP2 may have unique roles, especially in skeletal and cardiac muscles (6).

Overexpression of CAP1 has been found in cancers, including pancreatic, breast, ovarian, lung, esophageal, and liver cancers $(7,9,15-17)$. However, to date, only a few studies in hepatocellular and malignant melanoma have focused on CAP2 $(10,11,18)$. Shibata et al reported that CAP2 is upregulated in early $\mathrm{HCC}$ and even greater overexpression is observed in progressive HCC. They believe that the functional link between mitogen-activated protein kinase and cyclic AMP might be related to proliferative activity and carcinogenesis through CAP2 overexpression in HCC (10). Masugi et al reported that CAP2 overexpression is a novel prognostic marker in malignant melanoma. CAP2 expression seems to increase stepwise during tumor progression. IHC analysis of CAP2 could be helpful for histological identification of highly aggressive components in melanoma tissues and for early detection of aggressive subpopulations in clinical melanomas (11).

In this study, we present new evidence that the upregulation of CAP2 is associated with poor prognosis in breast carcinoma patients with both early- and late-stage disease. Our results clearly showed that elevation of CAP2 protein expression was observed in all of the five breast cancer cell lines, while the expression in non-tumorous HMECs was relatively very low. Then, we found that breast cancer lesions displayed higher CAP2 expression at the mRNA and protein levels as compared with adjacent non-cancerous tissues. Thus, we consider that CAP2 is an important molecular marker of breast cancer and can facilitate precise diagnoses. At present the precise roles of CAP2 in human cancers are still obscure. Cancer cell motility and metastasis depend on cell migration, adhesion and morphological change. Therefore, dynamic actin cytoskeleton reorganization and remodeling obviously increases and CAP may play a determinant role in these cell processes $(19,20)$. CAP2 overexpression in breast cancer may 
Table II. Cox regression analysis of various prognostic parameters for all patients.

\begin{tabular}{|c|c|c|c|c|}
\hline \multirow[b]{2}{*}{ Factors } & \multicolumn{2}{|c|}{ Univariate } & \multicolumn{2}{|c|}{ Multivariate } \\
\hline & HR $(95 \% \mathrm{CI})$ & P-value & HR $(95 \% \mathrm{CI})$ & P-value \\
\hline \multicolumn{5}{|l|}{ Age (years) } \\
\hline$<60$ & Reference & & & \\
\hline$\geq 60$ & $1.259(0.626-2.531)$ & 0.518 & - & - \\
\hline \multicolumn{5}{|c|}{ Clinical stage } \\
\hline I & Reference & 0.045 & Reference & \\
\hline II & $2.612(0.349-19.573)$ & 0.350 & $3.199(0.422-24.240)$ & 0.26 \\
\hline III & $5.436(0.721-41.011)$ & 0.101 & $6.479(0.847-49.541)$ & 0.072 \\
\hline \multicolumn{5}{|c|}{ T classification } \\
\hline $\mathrm{T} 1$ & Reference & 0.296 & & \\
\hline $\mathrm{T} 2$ & $1.601(0.613-4.182)$ & 0.337 & - & - \\
\hline $\mathrm{T} 3$ & $2.682(0.776-9.269)$ & 0.119 & - & - \\
\hline \multicolumn{5}{|c|}{$\mathrm{N}$ classification } \\
\hline No & Reference & 0.123 & & \\
\hline $\mathrm{N} 1$ & $0.694(0.277-1.739)$ & 0.435 & - & - \\
\hline $\mathrm{N} 2$ & $1.695(0.759-3.784)$ & 0.198 & - & - \\
\hline N3 & $2.407(0.784-7.389)$ & 0.125 & - & - \\
\hline \multicolumn{5}{|c|}{ Differentiation } \\
\hline Well & Reference & 0.187 & & \\
\hline Moderate & $4.036(0.548-29.743)$ & 0.171 & - & - \\
\hline Poor & $6.255(0.782-50.025)$ & 0.084 & - & - \\
\hline \multicolumn{5}{|c|}{ ER expression } \\
\hline Negative & Reference & & Reference & \\
\hline Positive & $0.387(0.199-0.753)$ & 0.005 & $0.412(0.21-0.805)$ & 0.01 \\
\hline \multicolumn{5}{|c|}{ PR expression } \\
\hline Negative & Reference & & & \\
\hline Positive & $0.334(0.166-0.671)$ & 0.002 & - & - \\
\hline \multicolumn{5}{|c|}{ HER2 expression } \\
\hline Negative & Reference & & & \\
\hline Positive & $1.006(0.483-2.095)$ & 0.987 & - & - \\
\hline \multicolumn{5}{|c|}{ CAP2 expression } \\
\hline Low & Reference & & Reference & \\
\hline High & $4.375(2.236-8.562)$ & 0.001 & $4.821(2.442-9.518)$ & 0.001 \\
\hline
\end{tabular}

reflect the aberrant regulation of actin dynamics. However, in order to understand the precise signaling pathways of CAP2 in breast cancer further studies are needed.

We further analyzed the relationship between the expression of CAP 2 and clinical characteristics of patients with breast cancer. There was a significant correlation between CAP2 and PR expression levels. Meanwhile, there were no significant correlations between the expression of CAP2 protein and patient age, clinical stage, $\mathrm{T}$ classification, $\mathrm{N}$ classification, differentiation, ER expression levels or HER2.

Reports have confirmed the prognostic value of CAP2 in human cancers. Fu et al reported that in a large cohort of 520 patients with HCC, CAP2 expression was significantly associated with overall and disease-free survival, and CAP2 was as an independent factor for prognostic prediction (21). CAP2 overexpression was also found to be a novel prognostic marker in malignant melanoma (11). However, the prognostic implication of CAP 2 in breast cancer has not been investigated. In our study, patients in the high CAP2 expression group had a $45.95 \%$ cumulative 10 -year survival rate, which was significantly lower than that of patients with low CAP2 expression levels (83.15\%). Multivariate analysis revealed that CAP2 expression might be an independent prognostic indicator for OS in breast cancer patients (Table II). This finding indicates the possibility of using high expression levels of CAP2 as a predictor for prognosis and survival. Interestingly, subgroup 
A

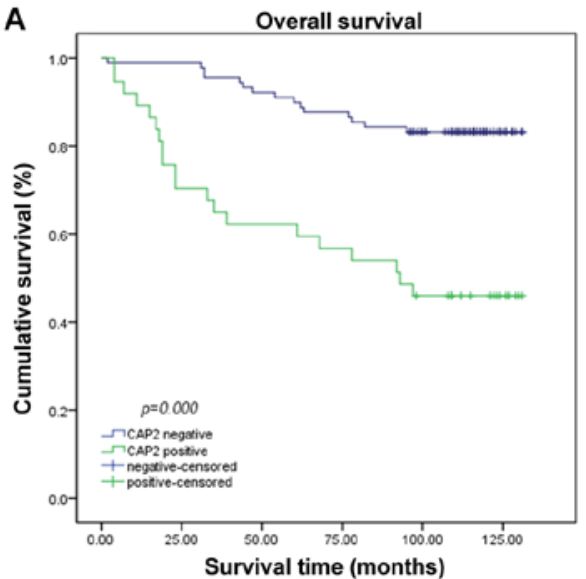

D Clinical stage I-II overall survival

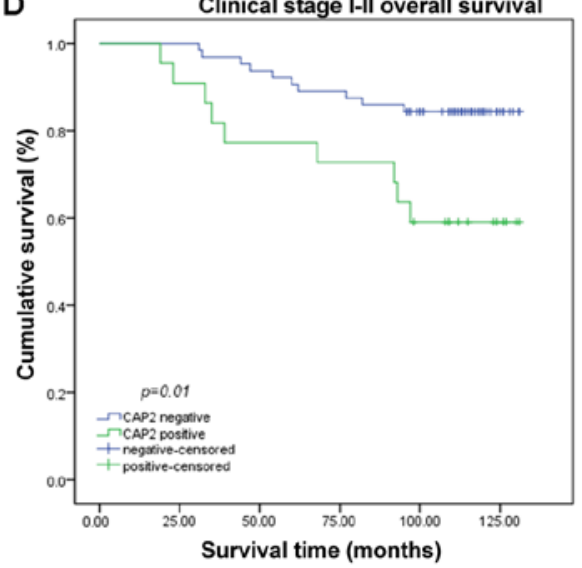

G

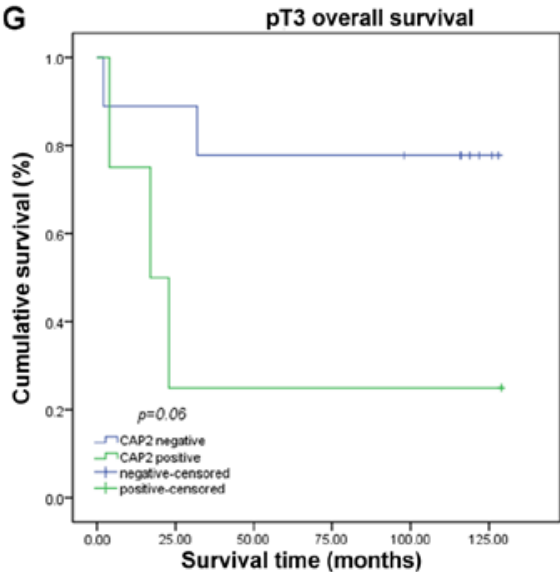

B
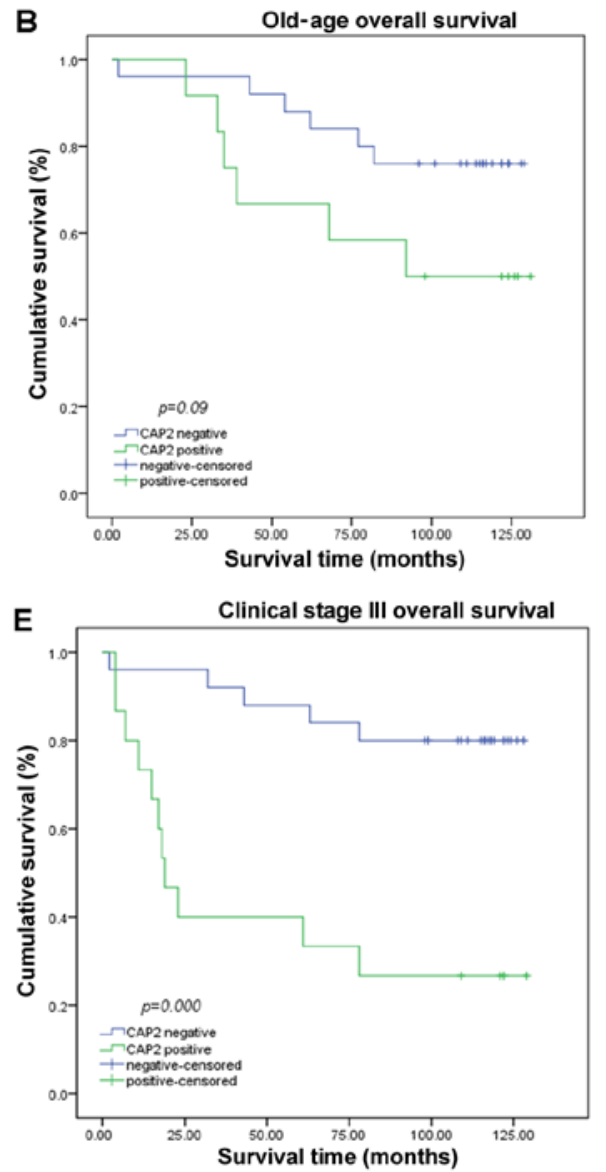

H

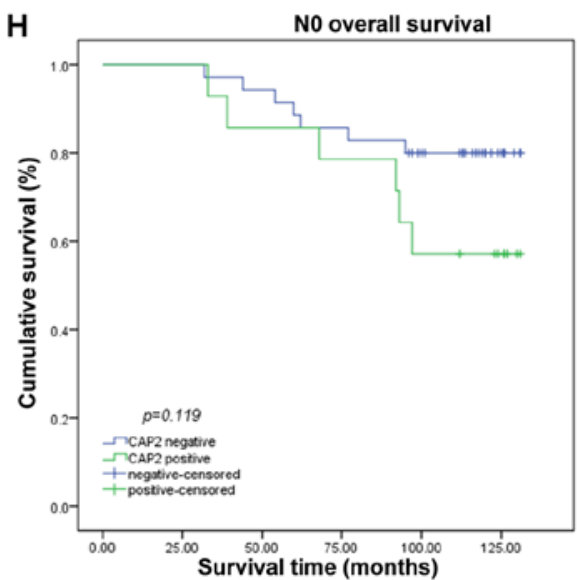

C

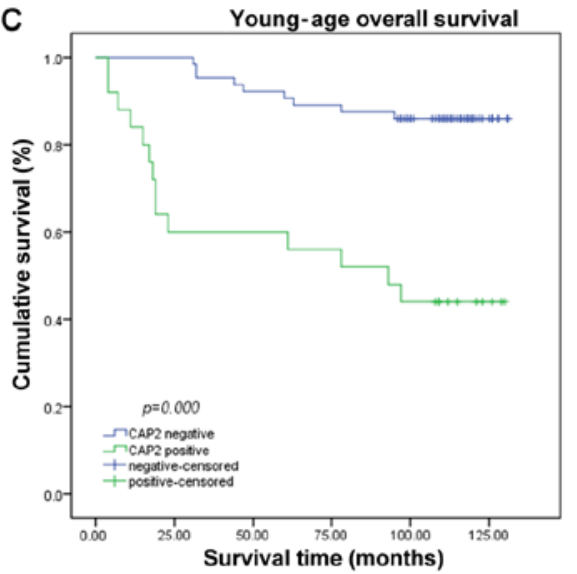

F

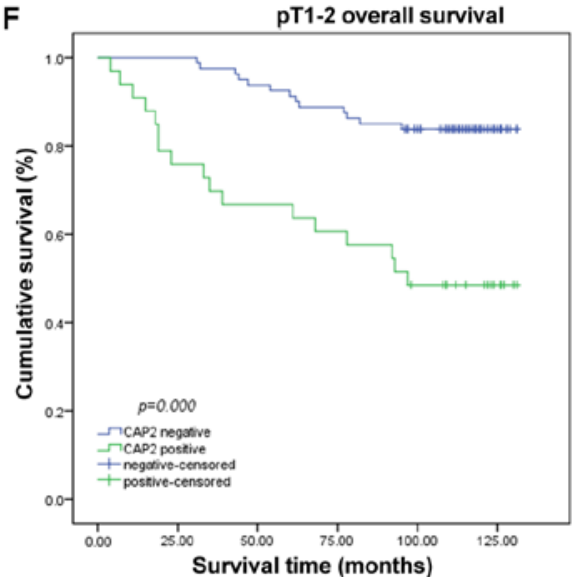

I

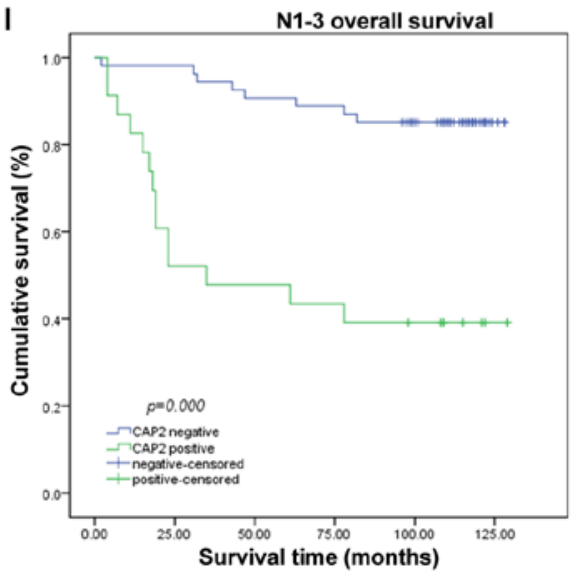

Figure 5. Kaplan-Meier curves with univariate analysis (log-rank). (A) OS rates for cases with high CAP2 expression vs. those with low CAP2 expression levels in all patients. (B) OS rates for old-age cases ( $\geq 60$ years) with high CAP2 expression vs. those with low CAP2 expression levels. (C) OS rates for young-age cases ( $<60$ years) with high CAP2 expression vs. those with low CAP2 expression levels. (D) OS rates for early clinical stage cases (stage I/II) with high CAP2 expression vs. those with low CAP2 expression levels. (E) OS rates for late-stage cases (stage III) with high CAP2 expression vs. those with low CAP2 expression levels. (F) OS rates for cases with high CAP2 expression vs. cases with low CAP2 expression levels in patients with T1-2-grade breast tumors. (G) OS rates for cases with high CAP2 expression vs. cases with low CAP2 expression levels in patients with T3-grade breast tumors. (H) OS rates for cases with high CAP2 expression vs. cases with low CAP2 expression levels in patients without lymphatic metastasis (N0). (I) OS rates for cases with high CAP2 expression vs. cases with low CAP2 expression levels in patients with lymphatic metastasis (N1-3). OS, overall survival; CAP2, cyclase-associated protein 2.

analysis revealed that CAP2-overexpression patients exhibited a significantly poor prognosis among patients whose tumors demonstrated the features of young age, early $\mathrm{T}$ stage and lymph node metastasis, respectively.

In conclusion, to the best of our knowledge, this is the first report addressing CAP2 expression and its clinicopathological and prognostic significance in breast cancer. Our findings suggest that CAP2 is upregulated in breast cancer and associated with the expression of PR. Multivariate analysis revealed that CAP2 might be an independent biomarker for the prediction of breast cancer prognosis and survival. Therefore, testing the CAP2 protein level may be helpful for stratifying patients for novel therapeutic strategy and establishing a rational treatment selection criteria for breast cancer patients. Further 
investigation is also needed to investigate the molecular mechanism of CAP2 involvement in the development and progression of breast cancer.

\section{Acknowledgements}

This study was supported by grants from the National Natural Science Foundation of China (81502268), the Guangdong Provincial Natural Science Foundation (2015A030313182, 2015A030310126), the Guangzhou Medical and Health Technology Program (20141A011075, 20151A011068), and Key Clinical Disciplines of Guangdong Province (20111219).

\section{References}

1. Torre LA, Bray F, Siegel RL, Ferlay J, Lortet-Tieulent J and Jemal A: Global cancer statistics, 2012. CA Cancer J Clin 65 $87-108,2015$

2. Makkonen M, Bertling E, Chebotareva NA, Baum J and Lappalainen P: Mammalian and malaria parasite cyclase-associated proteins catalyze nucleotide exchange on G-actin through a conserved mechanism. J Biol Chem 288: 984-994, 2013.

3. Fedor-Chaiken M, Deschenes RJ and Broach JR: SRV2, a gene required for RAS activation of adenylate cyclase in yeast. Cell 61: 329-340, 1990

4. Olson MF and Sahai E: The actin cytoskeleton in cancer cell motility. Clin Exp Metastasis 26: 273-287, 2009.

5. Yilmaz M and Christofori G: EMT, the cytoskeleton, and cancer cell invasion. Cancer Metastasis Rev 28: 15-33, 2009.

6. Peche V, ShekarS, Leichter M, Korte H, SchröderR, Schleicher M, Holak TA, Clemen CS, Ramanath-Y B, Pfitzer G, et al: CAP2 cyclase-associated protein 2 , is a dual compartment protein. Cell Mol Life Sci 64: 2702-2715, 2007.

7. Liu Y, Cui X, Hu B, Lu C, Huang X, Cai J, He S, Lv L, Cong X, Liu $\mathrm{G}$, et al: Upregulated expression of CAP1 is associated with tumor migration and metastasis in hepatocellular carcinoma. Pathol Res Pract 210: 169-175, 2014

8. Yu XF, Ni QC, Chen JP, Xu JF, Jiang Y, Yang SY, Ma J, Gu XL, Wang $\mathrm{H}$ and Wang $\mathrm{Y} Y$ : Knocking down the expression of adenylate cyclase-associated protein 1 inhibits the proliferation and migration of breast cancer cells. Exp Mol Pathol 96: 188-194, 2014.

9. Hua M, Yan S, Deng Y, Xi Q, Liu R, Yang S, Liu J, Tang C, Wang Y and Zhong J: CAP1 is overexpressed in human epithelial ovarian cancer and promotes cell proliferation. Int J Mol Med 35: 941-949, 2015.
10. Shibata R, Mori T, Du W, Chuma M, Gotoh M, Shimazu M, Ueda M, Hirohashi S and Sakamoto M: Overexpression of cyclase-associated protein 2 in multistage hepatocarcinogenesis. Clin Cancer Res 12: 5363-5368, 2006.

11. Masugi Y, Tanese K, Emoto K, Yamazaki K, Effendi K, Funakoshi T, Mori M and Sakamoto M: Overexpression of adenylate cyclase-associated protein 2 is a novel prognostic marker in malignant melanoma. Pathol Int 65: 627-634, 2015

12. Song LB, Zeng MS, Liao WT, Zhang L, Mo HY, Liu WL, Shao JY, Wu QL, Li MZ, Xia YF, et al: Bmi-1 is a novel molecular marker of nasopharyngeal carcinoma progression and immortalizes primary human nasopharyngeal epithelial cells. Cancer Res 66: 6225-6232, 2006.

13. Ono $\mathrm{S}$ : The role of cyclase-associated protein in regulating actin filament dynamics - more than a monomer-sequestration factor. J Cell Sci 126: 3249-3258, 2013.

14. Bertling E, Hotulainen P, Mattila PK, Matilainen T, Salminen M and Lappalainen P: Cyclase-associated protein 1 (CAP1) promotes cofilin-induced actin dynamics in mammalian nonmuscle cells. Mol Biol Cell 15: 2324-2334, 2004.

15. Yamazaki K, Takamura M, Masugi Y, Mori T, Du W, Hibi T, Hiraoka N, Ohta T, Ohki M, Hirohashi S, et al: Adenylate cyclase-associated protein 1 overexpressed in pancreatic cancers is involved in cancer cell motility. Lab Invest 89: 425-432, 2009.

16. Li M, Yang X, Shi H, Ren H, Chen X, Zhang S, Zhu J and Zhang J: Downregulated expression of the cyclase-associated protein 1 (CAP1) reduces migration in esophageal squamous cell carcinoma. Jpn J Clin Oncol 43: 856-864, 2013.

17. Xie SS, Tan M, Lin HY, Xu L, Shen CX, Yuan Q, Song XL and Wang $\mathrm{CH}$ : Overexpression of adenylate cyclase-associated protein 1 may predict brain metastasis in non-small cell lung cancer. Oncol Rep 33: 363-371, 2015.

18. Effendi K, Yamazaki K, Mori T, Masugi Y, Makino S and Sakamoto M: Involvement of hepatocellular carcinoma biomarker, cyclase-associated protein 2 in zebrafish body development and cancer progression. Exp Cell Res 319: 35-44, 2013.

19. Kirfel G, Rigort A, Borm B and Herzog V: Cell migration: Mechanisms of rear detachment and the formation of migration tracks. Eur J Cell Biol 83: 717-724, 2004.

20. Zhou GL, Zhang $H$ and Field J: Mammalian CAP (Cyclase-associated protein) in the world of cell migration: Roles in actin filament dynamics and beyond. Cell Adhes Migr 8: 55-59, 2014.

21. Fu J, Li M, Wu DC, Liu LL, Chen SL and Yun JP: Increased expression of CAP2 indicates poor prognosis in hepatocellular carcinoma. Transl Oncol 8: 400-406, 2015. 